\title{
CARACTERÍSTICAS DE CRESCIMENTO, DENSIDADE BÁSICA E COMPOSIÇÃO QUÍMICA DA MADEIRA DE Eucalyptus spp NA REGIÃO DE RIBAS DO RIO PARDO-MS
}

\section{BIOENG}

F.M.L. de Souza ${ }^{1 *}$, C.H. Pupo ${ }^{2}$, G.C. Sereghetti ${ }^{2}$, C.A. Sansígolo ${ }^{2}$, J.P. Ferreira $^{1}$, R.B. Silva ${ }^{1}$ e D.P. Garcia ${ }^{1}$

\author{
${ }^{1}$ FAIT - Faculdade de Ciências Sociais e Agrárias de Itapeva, Itapeva, SP, Brasil \\ ${ }^{2}$ UNESP - Universidade Estadual Paulista, Faculdade de Ciências Agronômicas de Botucatu, \\ Botucatu, SP, Brasil
}

Article history: Received 15 August 2017; Received in revised form 09 November 2017; Accepted 13 November 2017; Available online 27 December 2017.

\section{RESUMO}

O gênero Eucalyptus spp. se apresenta com uma qualidade potencial das mais interessantes, devido grande diversidade de espécies ligadas a uma boa capacidade produtiva e adaptação aos mais diversos tipos de clima e solo. Os plantios de clones, espécies e híbridos desse gênero são consolidados pela indústria de base florestal como a principal fonte de insumo nos processos de produção de celulose e papel. Nesse sentido, o presente trabalho objetivou avaliar os principais materiais genéticos em desenvolvimento no bioma cerrado com finalidade de caracterizar os parâmetros de crescimento, densidade básica e propriedades químicas da madeira na qualidade destas para a produção de celulose e papel. A pesquisa foi desenvolvida no ano de 2017 na Fazenda Jataí pertencente ao grupo Mutum, localizado no município de Ribas do Rio Pardo no Estado de Mato Grosso do Sul, sendo utilizadas oito espécies de Eucalyptus coletados em uma área clonal de espaçamento 3x3 metros com quatro anos de idade. As espécies utilizadas foram: Eucalyptus urophylla/ I220; E. urophylla I144; E. urophylla $\mathrm{x}$ E. grandis/ GG100; E. urophylla $\mathrm{x}$ E. grandis/ I224; E. urophylla $\mathrm{x}$ E. grandis/ VM19; E. urophylla x E. grandis/ I042; E. urophylla x E. camaldulensis / VM01 e E. camaldulensis / VM58. Os resultados mostraram que as densidades de seis materiais foram consideradas dentro do recomendado para as indústrias, o teor de holocelulose foi elevado em $50 \%$ dos materiais estudados e os teores de lignina e extrativos obtiveram baixos valores como quesito de qualidade para produção de celulose e papel. As características de crescimento também se mostraram viável para todas as espécies de Eucalyptus spp estudadas para a região de cerrado.

Palavras-chave: Crescimento volumétrico, lignina, holocelulose e extrativos.

\section{GROWTH CHARACTERISTICS, DENSITY BASIC AND CHEMICAL COMPOSITION OF WOOD Eucalyptus SPP IN THE REGION OF RIBAS DO RIO PARDO-MS}

\begin{abstract}
The Eucalyptus spp. is presented with a potential quality of the most interesting due to the great diversity of species linked to a good productive capacity and adaptation to the most diverse types of climate and soil. The clones, species and hybrids of this genus are consolidated by the forest-based industry as the main source of input in pulp and paper production processes. In this sense, the present work aimed to evaluate the main genetic material under development in the cerrado biome to characterize the parameters of growth,
\end{abstract}

\footnotetext{
* fabioleite@fait.edu.br
} 
basic density and chemical properties of the wood in the quality of these for pulp and paper production. The research was carried out in 2017 at Fazenda Jataí, belonging to the Mutum group, located in the municipality of Ribas do Rio Pardo in the State of Mato Grosso do Sul, using eight Eucalyptus species collected in a clonal area of $3 \times 3$ meters spacing with four years of age. The species used were: Eucalyptus urophylla / I220; E. urophylla / I144; E. urophylla $x$ E. grandis / GG100; E. urophylla x E. grandis / I224; E. urophylla x E. grandis / VM19; E. urophylla $x$ E. grandis / I042; E. urophylla $x$ E. camaldulensis / VM01 and E. camaldulensis / VM58. The results showed that the densities of six materials were considered within the industry recommended, the holocellulose content was elevated in $50 \%$ of the studied materials and the lignin and extractive contents obtained low values as quality for pulp and paper production. The growth characteristics were also shown to be feasible for all species of Eucalyptus spp studied for the cerrado region.

Keywords: volume growth, lignin, extractives, hemicellulose.

\section{INTRODUÇÃO}

A silvicultura brasileira vem se destacando com o crescente mercado de florestas plantadas. O país apresenta excelentes condições climáticas e amplas áreas para o desenvolvimento, além de possuir boas tecnologias no melhoramento genético de espécies florestais.

Segundo dados da Indústria Brasileira de Árvores- IBÁ (2015) o setor florestal brasileiro mantém hoje, em regime de produção, cerca de 7,74 milhões de hectares de áreas de florestas plantadas, sendo $72 \%$ do total, representado pelo gênero Eucalyptus. Os plantios de clones, espécies e híbridos desse gênero são consolidados pela indústria de base florestal como a principal fonte de insumo nos processos de produção de celulose e papel, carvão vegetal, painéis de madeira reconstituída e usinas de tratamento de madeira.

O que caracteriza o sucesso desse gênero no país são suas características fenológicas e a adaptação nas diferentes condições de clima e solo, fuste monopodial, rápido crescimento além de uma gama de uso de sua madeira pela indústria (SILVA \& XAVIER, 2006). Além disso, as inovações tecnológicas relacionadas às estratégias de melhoramento genético como a polinização controlada e a clonagem de genótipos superiores também são de suma importância, contribuindo para que $o$ Brasil alcançasse os mais elevados níveis de incremento médio anual (PALUDZYSZYN FILHO \& SANTOS, 2011).

Neste cenário, se destaca o Estado do Mato Grosso do Sul, que apresentou um crescimento da área florestal de $287 \%$ nos últimos sete anos, com uma área total aproximado de 803.699 ha, ficando atrás apenas dos Estados de São Paulo e Minas Gerais (IBÁ, 2015). O que proporcionou a crescente produção em Mato Grosso do Sul foram o apoio e incentivo do governo estadual, a possibilidade da diversificação de produtos nas propriedades rurais e a implantação de grandes empresas de base florestal na região.

Em 2013, a produção sul matogrossense de celulose alcançou 2,5 milhões de toneladas, perfazendo $20 \%$ da produção nacional (REFLORE, 2013). A expansão recente desse segmento tem fortalecido a importância do planejamento florestal no que diz respeito a qualidades das madeiras produzidas. A obtenção de padrões que relacionem qualidade da matéria-prima fornecida com o produto final na indústria é de suma importância, pois, apenas os valores fenotípicos das características dendrométricas não garante a seleção de genótipos com potencial tecnológico (BOTREL et al., 2010).

Com isso, este trabalho se apresenta oportuno para gerar maiores informações sobre o desenvolvimento e qualidade de oito diferentes materiais do gênero 
Eucalyptus spp, apresentando um estudo de crescimento e das propriedades físicas e químicas para a seleção de materiais

\section{MATERIAL E MÉTODOS}

A área experimental pertence ao Grupo Mutum, Fazenda Jataí, situada a 60 $\mathrm{Km}$ do município de Ribas do Rio Pardo no Estado de Mato Grosso do Sul, a uma altitude aproximada de 470 metros. O clima predominante na região segundo classificação de Koppen é o Aw, com médias de temperatura que variam entre $19^{\circ} \mathrm{C}$ e $23^{\circ} \mathrm{C}$. A precipitação média anual é de $1.200 \mathrm{~mm}$ a $1.500 \mathrm{~mm}$ anuais (CEMTEC, 2017).

Os materiais utilizados foram todos coletados em uma área de teste clonal (Tabela 1) no espaçamento $3 \times 3 \mathrm{~m}$ com 4 anos de idade.

Para o inventário florestal foi adotada a amostragem sistemática, em parcelas retangulares de 21 x 22 metros, totalizando $420 \mathrm{~m}^{2}$ onde foram levantados dados de diâmetro à altura do peito (DAP) e altura total de todos os indivíduos ocorrentes nas unidades amostrais. Para expressar o volume de madeira em função do diâmetro e altura, foi utilizado o modelo proposto por Schumacher \& Hall (1933). Todos os dados de inventário foram processados no software estatístico $\mathrm{R}$ ( $\mathrm{R}$ DEVELOPMENT CORE TEAM, 2011). superiores que visem atender o seguimento de celulose e papel.
Para a realização do trabalho foram abatidas 40 árvores (8 materiais $\mathrm{x} 5$ árvores) com DAP médio na população. Nas árvores abatidas foram retirados discos de $3 \mathrm{~cm}$ de espessura a $1,30 \mathrm{~m}$ do solo. $\mathrm{O}$ disco na altura do DAP representa uma forma de estimativa conforme trabalho de Trugilho (2009).

Os discos de madeira foram cortados em cunhas, sendo a primeira utilizada para a determinação da densidade básica (TAPPI T 258 om-94) e a segunda cunha para análise química, sendo esta reduzida a serragem em macro-moinho Wiley e classificada para obtenção da fração 40/60 mesh. Foram efetuadas as seguintes análises químicas em cada árvore: teor de extrativos totais (TAPPI T $264 \mathrm{~cm}-97$ ), lignina Klason insolúvel em ácido sulfúrico (TAPPI T 222 om-98) e holocelulose através da deslignificação com clorito de sódio, (ASTM 1104-56).

Inicialmente as variâncias foram testadas quanto à homogeneidade pelo teste de Barlett e posteriormente submetidas à análise de variância e aplicação de teste de médias Scott-Knott no nível de significância 5\%.

Tabela 1. Nomenclatura dos tratamentos das espécies de Eucalyptus spp utilizados na pesquisa em Ribas do Rio Pardo - MS (2017).

\begin{tabular}{cc}
\hline Tratamento & Espécies / nomenclatura no mercado \\
\hline $\mathrm{T} 1$ & Eucalyptus urophylla/ I220 \\
$\mathrm{T} 2$ & E. urophylla/ I144 \\
$\mathrm{T} 3$ & E. urophylla $\times$ E. grandis/ GG100 \\
$\mathrm{T} 4$ & E. urophylla $\mathrm{x}$ E. grandis/ I224 \\
$\mathrm{T} 5$ & E. urophylla $\times$ E. grandis/VM19 \\
$\mathrm{T} 6$ & E. urophylla $\times$ E. grandis/ I042 \\
$\mathrm{T} 7$ & E. urophylla $\mathrm{x}$ E. camaldulensis / VM01 \\
$\mathrm{T} 8$ & E. camaldulensis /VM58 \\
\hline
\end{tabular}




\section{RESULTADOS E DISCUSSÃO}

Incremento volumétrico e densidade básica das árvores

Observa-se na tabela 2 as características de crescimento das oito espécies em estudo, nota-se que a variação do incremento volumétrico ficou na faixa de 42,20 a 44,26 $\mathrm{m}^{3} \cdot \mathrm{ha}^{-1} \cdot$ ano $^{-1}$. Todos os tratamentos apresentaram valores acima da média ponderada pelas empresas associadas à Indústria Brasileira de Árvores - IBÁ (2015), que em 2014 atingiu $39,0 \mathrm{~m}^{3} \cdot \mathrm{ha}^{-1} \cdot$ ano $^{-1}$. Salienta-se que, a média de crescimento dos tratamentos foi superior em $9,6 \%$ à média nacional, com destaque para o hibrido de E. urophylla $x$ grandis -VM19 que apresentou o melhor desenvolvimento em volume (superior em $11,8 \%$ ). Verifica-se nas condições edafoclimáticas estudadas, uma maior adaptação e desenvolvimento dos materiais híbridos em comparação aos clones puros como E. urophylla - I220 e E. camaldulensis - VM58.

Com relação à estimativa de massa seca comercial, nota-se que a variável densidade básica, influenciou diretamente nos resultados finais de alguns materiais testados, como é o caso do E. urophylla $\mathrm{x}$ E. grandis/ I224 (alto volume e baixa massa seca) e E. urophylla/ I220 (baixo volume e alta massa seca). Estes resultados são importantes, pois demonstram que a seleção de materiais com base apenas no parâmetro volumétrico, não significam alta produção de massa seca que é um importante parâmetro na conversão final da quantidade de produtos a ser gerados pela indústria no processo de polpação.

Os valores médios obtidos de massa seca entre os materiais variaram de 59 a 72 $\mathrm{kg}$, numa comparação dos resultados com os trabalhos de Trugilho (2009) estudando cinco espécies de eucaliptos com a mesma idade e utilizando o mesmo método de amostragem e Trugilho et al., (2015) analisando a massa seca de madeira em clones e espécies de eucaliptos, verifica-se que todos os tratamentos apresentaram valores superiores ao reportado pelo autor demostrando a boa adaptação desses materiais a localidade.

Tabela 2. Valores médios das características de crescimento das espécies de Eucalyptus spp em: Diâmetro Altura do Peito, Altura Total, Incremento Médio Anual, Volume por hectare, Volume por Árvore e Massa Seca de Árvore em Ribas do Rio Pardo - MS (2017).

\begin{tabular}{|c|c|c|c|c|c|c|}
\hline Tratamento & $\begin{array}{l}\text { DAP } \\
(\mathrm{cm})\end{array}$ & $\begin{array}{l}\mathrm{HT} \\
(\mathrm{m})\end{array}$ & $\begin{array}{c}\text { IMA } \\
\left(\mathrm{m}^{3} \cdot \mathrm{ha}^{-}\right. \\
\left.{ }^{1} \cdot \text { ano }^{-1}\right) \\
\end{array}$ & $\begin{array}{c}\text { Volum } \\
\text { e/ha } \\
\left(\mathrm{m}^{3}\right)\end{array}$ & $\begin{array}{l}\text { Volume/ } \\
\text { árv }\left(\mathrm{m}^{3}\right)\end{array}$ & $\begin{array}{c}\text { Massa } \\
\text { seca } \\
(\mathrm{kg})\end{array}$ \\
\hline E. urophylla/ I220 & 16,0 & 19,82 & 42,20 & 168,8 & 0,153 & 69,05 \\
\hline E. urophylla/ I144 & 15,0 & 18,05 & 43,30 & 173,2 & 0,158 & 65,11 \\
\hline E.urophylla $\times$ E. grandis/ GG100 & 15,2 & 18,44 & 42,90 & 171,6 & 0,157 & 66,02 \\
\hline E. urophylla $\mathrm{x}$ E. grandis/ I 224 & 15,8 & 17,66 & 44,10 & 176,4 & 0,159 & 60,16 \\
\hline E. urophylla x E. grandis/VM19 & 15,4 & 18,62 & 44,26 & 177,0 & 0,161 & 72,10 \\
\hline E. urophylla x E. grandis/ I042 & 14,4 & 18,56 & 43,20 & 172,8 & 0,156 & 68,35 \\
\hline $\begin{array}{l}\text { E. urophylla x E. camaldulensis / } \\
\text { VM01 }\end{array}$ & 15,6 & 17,57 & 43,10 & 172,4 & 0,155 & 69,74 \\
\hline E. camaldulensis / VM58 & 17,0 & 16,10 & 42,30 & 169,2 & 0,154 & 59,57 \\
\hline
\end{tabular}

DAP = diâmetro a 1,3 m de altura do solo; HT = altura total; IMA = Incremento médio anual; Volume por hectare; Volume por árvore e Massa seca média árvore.

Em relação à densidade básica (Tabela 3), nota-se primeiramente na classe dos híbridos de E. urophylla $x E$. grandis uma variação significativa da densidade básica com valores na faixa de $0,376 \mathrm{~g} \mathrm{~cm}^{-3}$ a $0,451 \mathrm{~g} \mathrm{~cm}^{-3}$. Alves et al., 
(2014), avaliando a densidade de cinco híbridos de E. grandis $x$ E. urophylla na altura do DAP e mesma idade obtiveram valores entre $0,457 \mathrm{~g} \mathrm{~cm}^{-3}$ e $0,533 \mathrm{~g} \mathrm{~cm}^{-3}$, acima dos encontrados no presente estudo.

Outra diferença significativa observada foi à variação entre $\mathrm{o} \quad E$. urophylla / I220 com o E. camaldulensis/ VM58 que reforça a importância de híbridos no aumento do volume e da densidade básica como constatado no hibrido de E. urophylla $x$ E. camaldulensis/ VM01.

Tabela 3. Valores médios da densidade básica da madeira, Desvio Padrão e Coeficiente de variação das espécies de Eucalyptus spp em Ribas do Rio Pardo - MS (2017).

\begin{tabular}{cccc}
\hline Tratamento & $\begin{array}{c}\text { Densidade básica } \\
\left(\mathrm{g} \mathrm{cm}^{-3}\right)\end{array}$ & $\begin{array}{c}\text { Desvio } \\
\text { Padrão } \\
\left(\mathrm{g} \mathrm{cm}^{-3}\right)\end{array}$ & $\begin{array}{c}\text { Coeficiente de } \\
\text { variação } \\
(\%)\end{array}$ \\
\hline E. urophylla/ I220 & $0,450^{\mathrm{a}}$ & 0,04 & 10,59 \\
E. urophylla/ I144 & $0,414^{\mathrm{b}}$ & 0,00 & 1,46 \\
E.urophylla $\times$ E. grandis/ GG100 & $0,427^{\mathrm{a}}$ & 0,00 & 1,80 \\
E. urophylla X E. grandis/ I224 & $0,376^{\mathrm{b}}$ & 0,01 & 4,15 \\
E. urophylla $\mathrm{x}$ E. grandis/ VM19 & $0,451^{\mathrm{a}}$ & 0,01 & 3,02 \\
E. urophylla $\mathrm{x}$ E. grandis/ I042 & $0,439^{\mathrm{a}}$ & 0,01 & 2,63 \\
E. urophylla $\mathrm{x}$ E. camaldulensis / & & & 3,41 \\
VM01 & $0,448^{\mathrm{a}}$ & 0,01 & 2,50 \\
\hline E. camaldulensis / VM58 & $0,389^{\mathrm{b}}$ & 0,01 &
\end{tabular}

Médias seguidas de mesma letra na coluna não diferem significativamente pela aplicação de teste de médias Scott-Knott a 5\% de probabilidade.

A faixa de densidade básica limita a escolha do material genético de acordo com a finalidade, além de apresentar certa relevância na padronização da matéria prima recebida pela indústria. Na produção de celulose de fibra curta, a densidade básica recomendada varia de $0,400 \mathrm{~g} \mathrm{~cm}^{-3}$ a $0,550 \mathrm{~g} \mathrm{~cm}^{-3}$, o que pode ser observado em seis dos oito materiais estudados. Em geral, madeiras com baixa densidades

Análise da composição química dos materiais

Referente à composição química das madeiras, observa-se para a variável holocelulose, diferenças significativas entre os materiais. Em geral, é possível constatar a divisão entre dois grupos, com valores médios significativamente superiores na faixa de $77 \%$ para $E$. urophyllal $\mathrm{I} 220, E$. urophylla $\mathrm{x} E$. grandis/ VM19, E. urophylla $\times$ E. grandis/ I042 e E. camaldulensis / VM58. O menor resultado foi observado para o hibrido de E. urophylla x E. camaldulensis / VM01 com $73,98 \%$. levam à redução do rendimento volumétrico em celulose, enquanto as madeiras com maior densidade apresentam dificuldades de picagem e impregnação de cavacos, consomem mais reagentes $\mathrm{e}$ levam a baixos rendimentos gravimétricos e teores mais elevados de rejeitos (QUEIROZ et al., 2004; TRUGILHO et al., 2005; SANTOS \& SANSÍGOLO, 2007)

O termo holocelulose é designado na representação dos polissacarídeos totais presentes na madeira, e o seu elevado teor é diretamente proporcional ao maior rendimento nos processos de polpação. De forma geral, independente das diferenças significativas é possível observar que os valores obtidos foram superiores aos trabalhos de Santana et al., (2012) que reportam valores de $68,13 \%$ e Trugilho et al., (2015) com valores médios de 61,90\%. Em ambos os trabalhos os autores calcularam o teor de holocelulose por 
diferença (holocelulose $=100-$ extrativos totais - lignina total). Para trabalhos que utilizaram o mesmo procedimento como o de Trugilho et al., (2005) analisando 15 híbridos de Eucalyptus spp., o autor destaca valores médios de 76,46\%, e de $77,8 \%$ para o estudo de Sansígolo \& Curvelo (1994) analisando o Eucalyptus globulus, valores próximos ao encontrado neste estudo mesmo com idades superiores. O que é explicado pelo trabalho de Santana et al., (2012) que reporta em sua pesquisa que os teores de holocelulose não foram afetados pela idade e nem pela classe de diâmetro.

Tabela 4. Porcentagem do teor de holocelulose, Desvio padrão e Coeficiente de variação das espécies de Eucalyptus spp em Ribas do Rio Pardo - MS (2017).

\begin{tabular}{cccc} 
Tratamento & $\begin{array}{c}\text { Teor de } \\
\text { holocelulose } \\
(\%)\end{array}$ & $\begin{array}{c}\text { Desvio } \\
\text { Padrão } \\
(\%)\end{array}$ & $\begin{array}{c}\text { Coeficiente de } \\
\text { variação } \\
(\%)\end{array}$ \\
\hline E. urophylla/ I220 & $77,60^{\mathrm{a}}$ & 1,80 & 2,42 \\
E. urophylla/ I144 & $74,37^{\mathrm{b}}$ & 1,69 & 2,28 \\
E.urophylla $\mathrm{x}$ E. grandis/ GG100 & $74,53^{\mathrm{b}}$ & 2,28 & 3,03 \\
E. urophylla $\times$ E. grandis/ I224 & $75,35^{\mathrm{b}}$ & 2,00 & 2,71 \\
E. urophylla $\mathrm{x}$ E. grandis/ VM19 & $77,03^{\mathrm{a}}$ & 0,77 & 0,96 \\
E. urophylla $\mathrm{x}$ E. grandis/ I042 & $77,81^{\mathrm{a}}$ & 1,14 & 1,48 \\
E. urophylla $\mathrm{x}$ E. camaldulensis / & & & 1,97 \\
VM01 & $73,98^{\mathrm{b}}$ & 1,53 & 1,26 \\
\hline E. camaldulensis /VM58 & $77,26^{\mathrm{a}}$ & 0,97 &
\end{tabular}

Médias seguidas de mesma letra na coluna não diferem significativamente pela aplicação de teste de médias Scott-Knott a 5\% de probabilidade.

Em relação aos resultados médios do teor de lignina (Tabela 5), observa-se que não houve diferenças significativas entre os tratamentos. Nota-se que os híbridos apresentaram menor variação entre médias, com valores na faixa de $24 \%$ quando comparado com as espécies puras de $E$. urophylla /I220 que apresentou 23,07\% e E. camaldulensis / VM58 com 25,83\%. Além disso, as diferenças significativas da densidade básica não afetaram no comportamento dos teores de lignina. Contudo, Silva et al., (2005) e Protasio et al., (2014), relatam a influência da idade na composição química da madeira, de modo que a lignina aumente com o passar dos anos, com a tendência inversa para holocelulose.
Referente aos materiais deste estudo quando comparado a resultados de outros autores, nota-se que são inferiores, pelo fato que neste trabalho foi avaliado apenas a lignina insolúvel enquanto na literatura avaliaram-se a lignina total. A exemplo, Trugilho (2009), analisando E. urophylla e E. grandis com a mesma idade encontrou valores variando de $27,61 \%$ a $28,04 \%$. Neves et al., (2011), avaliando o clone I144 (T2) e o clone I220 (T1) em diferentes regiões no Estado de Minas Gerais encontraram valores na faixa de $27,86 \%$ a $31,58 \%$. Trugilho et al., (2015), analisando híbridos de E. grandis x $E$. urophylla também com 4 anos de idade obtiveram valores variando de $29,72 \%$ a $31,31 \%$. 
Tabela 5. Porcentagem do teor de Lignina, Desvio padrão e Coeficiente de variação das espécies de Eucalyptus spp em Ribas do Rio Pardo - MS (2017).

\begin{tabular}{cccc}
\hline Tratamento & $\begin{array}{c}\text { Teor de lignina } \\
(\%)\end{array}$ & $\begin{array}{c}\text { Desvio } \\
\text { Padrão } \\
(\%)\end{array}$ & $\begin{array}{c}\text { Coeficiente de } \\
\text { variação } \\
(\%)\end{array}$ \\
\hline E. urophylla/ I220 & $23,07^{\mathrm{a}}$ & 1,04 & 4,22 \\
E. urophylla/ I144 & $24,82^{\mathrm{a}}$ & 0,95 & 3,97 \\
E.urophylla $\mathrm{x}$ E. grandis/ GG100 & $24,00^{\mathrm{a}}$ & 1,45 & 6,54 \\
E. urophylla $\times$ E. grandis/ I224 & $22,30^{\mathrm{a}}$ & 0,33 & 1,38 \\
E. urophylla $\times$ E. grandis/ VM19 & $24,36^{\mathrm{a}}$ & 1,22 & 6,75 \\
E. urophylla $\times$ E. grandis/ I042 & $24,13^{\mathrm{a}}$ & 1,05 & 4,56 \\
E. urophylla $\times$ E. camaldulensis / & & & \\
VM01 & $24,43^{\mathrm{a}}$ & 2,29 & 9,50 \\
E. camaldulensis / VM58 & $25,83^{\mathrm{a}}$ & 1,56 & 6,04 \\
\hline
\end{tabular}

Médias seguidas de mesma letra na coluna não diferem significativamente pela aplicação de teste de médias Scoott-Knott a 5\% de probabilidade.

Como na produção de celulose por meio de processos químicos, a lignina é considerada um constituinte prejudicial. Altos teores nas madeiras demandam mais álcali ativo no processo de impregnação dos cavacos durantes as operações de cozimentos e branqueamento, prejudicando a operação (CARDOSO et al., 2002; TRUGILHO et al., 2005; GOMIDE et al., 2005; FERREIRA et al., 2006; SANTOS \& SANSÍGOLO, 2007).

Por fim, o resultado médio do teor de extrativos apresentado na Tabela 6 , mostra que houve diferença significativa entre os tratamentos com destaque para o hibrido de E. urophylla x E. grandis/ VM19 com $0,80 \%$. Os maiores resultados foram observados no E.urophylla x E. grandis/ GG100 e E. Camaldulensis / VM01 com valores acima de 2,0\%. Foelkel (2009) relata que os valores mais comuns de extrativos podem variar de $1,5 \%$ até $6 \%$. Os baixos valores encontrados neste estudo pode estar relacionando com a idade do material como verificado por Silva et al., (2005) estudando E. grandis com quatro idades distintas. Contudo, os resultados mostram-se importantes, pelo fato de altos teores de extrativos serem compostos indesejáveis, por dificultarem a impregnação dos cavacos além de reduzir o rendimento e prejudicar a qualidade da polpa (FERREIRA et al., 2006; WEHR \& BARRICHELO, 1992).

Tabela 6. Porcentagem do teor de extrativos, Desvio padrão e Coeficiente de variação das espécies de Eucaliptos spp. em Ribas do Rio Pardo - MS (2017).

\begin{tabular}{cccc} 
Tratamento & $\begin{array}{c}\text { Teor de } \\
\text { extrativos } \\
(\%)\end{array}$ & $\begin{array}{c}\text { Desvio } \\
\text { Padrão } \\
(\%)\end{array}$ & $\begin{array}{c}\text { Coeficiente } \\
\text { de variação } \\
(\%)\end{array}$ \\
\hline E. urophylla/ I220 & $1,30^{\mathrm{b}}$ & 0,57 & 33,69 \\
E. urophylla/ I144 & $1,39^{\mathrm{b}}$ & 0,41 & 29,63 \\
E.urophylla $\mathrm{x}$ E. grandis/ GG100 & $2,16^{\mathrm{c}}$ & 0,37 & 17,17 \\
E. urophylla $\mathrm{x}$ E. grandis/ I224 & $1,74^{\mathrm{c}}$ & 0,64 & 37,29 \\
E. urophylla $\mathrm{x}$ E. grandis/ VM19 & $0,80^{\mathrm{a}}$ & 0,44 & 34,15 \\
E. urophylla $\mathrm{x}$ E. grandis/ I042 & $1,21^{\mathrm{b}}$ & 0,08 & 10,30 \\
E. urophylla $\mathrm{x}$ E. camaldulensis / VM01 & $1,70^{\mathrm{c}}$ & 0,57 & 33,69 \\
E. camaldulensis /VM58 & $2,19^{\mathrm{c}}$ & 0,51 & 23,67 \\
\hline
\end{tabular}

Médias seguidas de mesma letra na coluna não diferem significativamente pela aplicação de teste de médias Scott-Knott a 5\% de probabilidade. 
O estado do Mato Grosso do Sul por apresentar uma área de reflorestamento ainda muito recente, pouco se conhece sobre as influências das condições edafoclimáticas do local e como essas podem influenciar nas propriedades físicas, químicas e anatômicas da madeira. Com isso, é possível que essas condições, juntamente com o manejo silvicultural tenha possibilitado os bons resultados

\section{CONCLUSÕES}

A produtividade média anual e a massa seca foram elevadas em todos os tratamentos destacando a adaptação edafoclimática dos clones para região de Ribas do Rio Pardo-MS.

A densidade básica e as análises químicas possibilitaram concluir que os materiais deste estudo apresentam alto potencial para produção de celulose e papel, constando que as densidades de seis materiais foram consideradas dentro do recomendado para as indústrias.

\section{REFERÊNCIAS}

ALVES, A. P. L. A.; PAULA N. F. de.; PAULA R. C. de.; Wood density in clones of hybrids eucalyptus. Ciência \& Tecnologia: Fatec-JB, Jaboticabal, v. 6, p. 183-186, 2014.

ASTM D1104-56. Method of test for holocellulose in wood. 1978. http://www.astm.org/Standards/D1104.htm

BOTREL, M. C. G.; TRUGILHO, P. F.; ROSADO, S.C.S.; MOREIRA DE SILVA, J.R.; Seleção de clones de Eucalyptus para biomassa florestal e qualidade da madeira. Scientia Forestalis, v. 38 , n. 86 , p. 237245, 2010.

CARDOSO, G. V.; ROSA, C. A. B.; GUARIENTI, A. F.; PEDRAZZI, C.; SOUZA, M. C. H.; FRIZZO, S. M. B.; FOELKEL, C. E.B. Adequação de metodologias amostral de madeira de Eucalyptus saligna e Eucalyptus glóbulos apresentados neste estudo, como maiores teores de polissacarídeos e menores teores de lignina e extrativos que diretamente propiciam no melhor desempenho da qualidade da polpa em termos de rendimento final de celulose (QUEIROZ et al., 2004; TRUGILHO et al., 2005; FERREIRA et al., 2006; SANTOS \& SANSÍGOLO, 2007; GOUVÊA et al., 2009).

O teor de holocelulose foi elevado em $50 \%$ dos materiais estudados e os teores de lignina e extrativos considerados baixos em todos os tratamentos.

O hibrido de E. urophylla x $E$. grandis/ VM19 por apresentar o maior volume, a maior densidade básica e o menor teor de lignina, foi o destaque, considerando o seu uso em processos de polpação.

para determinação do teor de cinzas. In: CONGRESSO INTERNACIONAL DE CELULOSE E PAPEL, 34., 2001, São Paulo. Anais... São Paulo: Associação Brasileira Técnica de Celulose e Papel, 2001. p. 7.

Centro de monitoramento do tempo, clima e dos recursos hídricos do Mato Grosso do Sul. CEMTEC/MS-AGRAER. Disponível em: http://www.cemtec.ms.gov.br/page_id=15. Acesso em: 29/10/2017

FOELKEL, C. Propriedades papeleiras das árvores, madeiras e fibras celulósicas dos Eucaliptos. Eucalyptus Online Book \& Newsletter.111p. 2009. Disponível em: <http://www.eucalyptus.com.br/disponivei s.html>. Acesso em: 25 maio. 2017.

FERREIRA, C. R.; FANTINI JUNIOR, M.; COLODETTE, J. L.; GOMIDE, J. L.; 
CARVALHO, A. M. L.; Avaliação tecnológica de clones de eucalipto: parte 1qualidade da madeira para produção de celulose Kraft. Scientia Forestalis, Piracicaba, n. 70, p. 161-170, abr. 2006.

GOMIDE, J. L.; FANTUZZI NETO, H.; REGAZZI, A. J.; Caracterização tecnológica, para produção de celulose, da nova geração de clones de eucalipto no Brasil. Revista Árvore, Viçosa, MG, v. 29, n. 1, p. 129-137, 2005.

GOUVÊA, A. F. G.; TRUGILHO, P. F.; COLODETTE, J. L.; LIMA, J. T.; SILVA, J. R. M.; GOMIDE, J. L.; Avaliação da madeira e da polpação Kraft em clones de Eucaliptos. Revista Árvore, Viçosa, MG, v. 33, n. 6, p.1175-1185, 2009.

IBÁ - Indústria Brasileira de Árvores. Indicadores do setor brasileiro de árvores plantadas 2015: ano base 2014. Brasília, p.49-65, 2015 Disponível em < http://www.iba.org/pt>. Acesso em: 14 de agosto 2017.

NEVES, T. A.; PROTÁSIO, T. P.; COUTO, A. M.; TRUGILHO, P. F.; SILVA, V. O.; VIEIRA, C. M. M.; Avaliação de clones de Eucalyptus em diferentes locais visando à produção de carvão vegetal. Pesquisa Florestal Brasileira, Colombo, v. 31, n. 68, p. 319 330, 2011.

PALUDZYSZYN FILHO, E.; SANTOS, P. E. T.; Programa de melhoramento genético de eucalipto da Embrapa Florestas: resultados e perspectivas. Colombo: Embrapa Florestas, 2011. 66 p. (Embrapa Florestas. Documentos, 2014).

PROTÁSIO, T. P.; NEVES, T. A.; REIS, A. A. dos; TRUGILHO, P. F.; Efeito da idade e clone na qualidade da madeira de Eucalyptus spp. visando à produção de bioenergia. Ciência Florestal, Santa Maria, v. 24, n.2, p. 465-477, 2014.
QUEIROZ, S. C. S.; GOMIDE, J. L.; COLODETTE, J. L.; OLIVEIRA, R. C.; Influência da densidade básica da madeira na qualidade da polpa kraft de clones híbridos de Eucalyptus grandis W. Hill ex Maiden x Eucalyptus urophylla S.T. Blake clones. Revista Árvore, Viçosa, MG, v. 28, n. 6, p. 901-909, 2004.

R Development Core Team. R: A language and environment for statistical computing. R Foundation for Statistical Computing, Vienna, Austria, 2011.

REFLORE, Informativo da Associação Sul-mato-grossense de Produtores e Consumidores de Florestas Plantadas, MS Florestal, 2013.

SANTANA, W.M.S.; CALEGARIO, N.; ARANTES, M. D. C.; TRUGILHO, P. F.; Effect of age and diameter class on the propperties of wood from clonal Eucalyptus. Cerne, Lavras, v. 18, n. 1, p. 1-8, jan./mar. 2012

SANTOS, S. R.; SANSÍGOLO, C. A.; Influência da densidade básica da madeira de clones de Eucalyptus grandis $\mathrm{x}$ Eucalyptus urophylla na qualidade da polpa branqueada. Ciência Florestal, Santa Maria, v. 17, n.1, p. 53-63, jan./mar. 2007.

SANSÍGOLO, C. A.; CURVELO, A. A. S.; Deslignificação em etanol-água de Eucalyptus globulus: características da lignina e da polpa. O Papel, São Paulo, p. 23-29, ago. 1994.

SCHUMACHER, F. X.; HALL, F. S.; Logarithmic expression of timber-tree volume. Journal of Agricultural Research, v.47, n.9, p.719-734, 1933.

SILVA, J. C.; MATOS, J. L. M.; OLIVEIRA, J. T. S.; EVANGELISTA, W. V.; Influência da idade e da posição ao longo do tronco na composição química da madeira de Eucalyptus grandis Hill ex. Maiden. Revista Árvore, Viçosa, MG, v. 29, n. 3, p. 455-460, 2005. 
SILVA, J. C.; XAVIER, B. A.; Eucalipto: manual prático do fazendeiro florestal, produzindo madeira com qualidade. Viçosa, MG, 2006. 65 p.

TAPPI T 222 om-98, Acid-insoluble lignin in wood and pulp. In: Test methods 1983 - 1998. TAPPI Press, Atlanta, USA.

TAPPI T 258 om-94, 1994. Basic Density and Moisture content of pulpwood. In: Test Methods 1985 - 1994. TAPPI Press, Atlanta, USA.

TAPPI T $264 \mathrm{~cm}-97$, Preparation of wood for chemical analysis. In: Test methods 1982 - 1997. TAPPI Press, Atlanta, USA.

TRUGILHO, P. F.; BIANCHI, M. L.; GOMIDE, J. L.; LIMA, J. T.; MENDES, L. M.; MORI, F. A.; GOMES, D. F. F.; Clones de Eucalyptus versus a produção de polpa celulósica. Ciência Florestal, Santa Maria, v. 15, n. 2, p. 145-155, 2005.
TRUGILHO, P. F.; Basic density and dry mass and lignin mass estimate in Eucalyptus wood species. Ciência e Agrotecnologia, Lavras, v. 33, n. 5, p. 1228-1239, 2009.

TRUGILHO, P. F.; GOULART, S. L.; ASSIS, C. O. de; COUTO, F. B. S.; ALVES, I. C. N.; PROTÁSIO, T. P.; NAPOLI, A.; Growing characteristics, chemical composition, physical and dry mass estimated of wood in young Eucalyptus species and clones. Ciência Rural, Santa Maria, v.45, n.4, p.661-666, 2015.

WEHR, T. R.; BARRICHELO, L. E. G.; Cozimento kraft com madeiras de Eucalyptus grandis de diferentes densidades básicas e dimensões de cavacos. In: CONGRESSO ANUAL DE CELULOSE E PAPEL, 25., São Paulo, 1992. Anais...São Paulo: ABTCP, 1992. p.161-177. v. 25, p. 1-17. 